\title{
Dilthey, Heidegger und die Hermeneutik des faktischen Lebens
}

\section{Einleitung ${ }^{1}$}

Die Begriffsbildungen Endlichkeit, Faktizität, Geschichtlichkeit und Hermeneutik, die sich alle auf das Verstehen des menschlichen Daseins beziehen, werden verständlicherweise in der gegenwärtigen Philosophie häufig in Verbindung mit Martin Heideggers frühem philosophischem Projekt gebracht und von hier aus auch gedeutet. Weniger wird jedoch darauf geachtet, wie stark Heidegger diese leitenden Grundbegriffe mit Bezug auf Wilhelm Diltheys Denken über die menschliche Existenz interpretiert und auf seine eigene Art und Weise umgewandelt hat. ${ }^{2}$

Im Zusammenhang der wichtigen Auseinandersetzung des frühen Heidegger mit Dilthey in den 1920er Jahren hat Heidegger Edmund Husserls transzendentale Phänomenologie erst lebensphilosophisch und dann hermeneutisch umgedeutet. Heidegger hat in diesem Kontext die »kategoriale Anschauung" (die nach Husserl das Universale im Singularen wahrnimmt) als eine interpretative Hermeneutik und als »formal anzeigend « gedeutet; eine Formalisierung, die auf das "auslegende Vorlaufen« der konkreten Faktizität des menschlichen Daseins in seiner Mannigfaltigkeit hinweist. ${ }^{3}$

Nach Diltheys Begriff einer »modernen Lebensphilosophie« kann das Leben

1 Ich möchte Malgorzata Bogaczyk-Vormayr, Annette Hilt und Gunter Scholtz besonders danken sowie den Teilnehmern der Breslauer Dilthey-Tagung vom Oktober 2011.

2 Vgl. Jae-Chul Kim, Leben und Dasein: die Bedeutung Wilhelm Diltheys für den Denkweg Martin Heideggers, Würzburg 2001.

3 Die Hermeneutik der Faktizität, als formal anzeigend verstanden, ist bei Heidegger mit und gegen Dilthey entwickelt und umgestaltet. Vgl. Martin Heidegger, Phänomenologie der Anschauung und des Ausdrucks, GA (= Gesamtausgabe) Bd. 59, Frankfurt a. M. 1993, S. 154, 167. Vgl. weiter meine Deutung von Heideggers Methode der Formalisierung in: Eric S. Nelson, Questioning Practice: Heidegger, Historicity and the Hermeneutics of Facticity. In: Philosophy Today 44 (2001), S. 150 - 159; ebenso E. S. Nelson, Die formale Anzeige der Faktizität als Frage der Logik. In: Heidegger und die Logik, hg. von Alfred Denker, Holger Zaborowski, Amsterdam: Editions Rodopi BV, 2006, S. $31-48$. 
nur "aus ihm selbst gedeutet werden. $\aleph^{4}$ Die eindrucksvollen, sehr frei ausgedrückten und gefühlsmäßigen Deutungen des Lebensbegriffs, die von den Lebensphilosophen stammen, sollen von einer in der Luft schwebenden Spekulation befreit werden und zu wahrer Erkenntnis führen. Das gelingt laut Dilthey, wenn diese Deutungen in der Selbstbesinnung und in der wissenschaftlichen Reflexion methodisch und begrifflich geklärt werden. Heidegger hat teilweise und zwar in seinen früheren Vorlesungen nach dem Ersten Weltkrieg - in ähnlicher Weise die Philosophie als einen Versuch dargestellt, das faktische Leben aus sich selbst zu verstehen. ${ }^{5}$ Aber das "Aus-sich-selbst Verstehen « ist beim früheren Heidegger gegen die erkenntnistheoretischen und psychologischen Tendenzen der neuzeitlichen Philosophie gewendet, die er immer noch im Neukantianismus sowie bei Husserl und Dilthey spürt und beklagt und die er besonders in der Mitte der 1920er Jahre im Rückgriff auf Aristotelus gedeutet hat. $^{6}$

\section{Das Faktische Leben und die Formale Anzeige}

Das »Sich-selbst-Verstehen« heißt, das faktische Leben zu Wort (»logos») kommen zu lassen. D.h. dieses Leben »spricht mit sich selbst« (»kategorein«), und in diesem Gespräch kommt das Leben zum immanenten »kategorialen" Sinn des faktischen Lebens ${ }^{7}$ : "Das Wie [philosophischer] Forschung ist die Interpretation dieses Seinssinnes auf seine kategorialen Grundstrukturen: das heißt die Weisen, in denen faktisches Leben sich selbst zeitigt und zeitigend mit sich selbst spricht (kategorein). ${ }^{8}$ Die Sprache selbst hebt an als Ansprechen von Welt, so wie die Welt sich zeigt: "Das ursprüngliche Wort war eine Nennung, aber nicht eines bloßen Namens; vielmehr etwas, was in der Welt begegnet, wird angesprochen, wie es begegnet. " 9

Die Begegnung selbst ist zugleich ein Ansprechen und Angesprochensein.

4 Dilthey, Das Wesen der Philosophie (1909), GS Bd. 5, S. 370.

5 Vgl. Georg Misch, Lebensphilosophie und Phänomenologie. Eine Auseinandersetzung der Diltheyschen Richtung mit Heidegger und Husserl, Darmstadt 1967, S. 7.

6 Vgl. Martin Heidegger, Phänomenologische Interpretationen zu Aristoteles: Einführung in die phänomenologische Forschung, GA Bd.61, hg. Walter Bröcker, Käte Bröcker-Oltmanns, Frankfurt a. M. 1985; Martin Heidegger, Phänomenologische Interpretationen zu Aristoteles, Stuttgart 2003 (im Folgenden wird diese Ausgabe zitiert).

7 Martin Heidegger, Phänomenologische Interpretationen, S. 28.

8 Ebd., S. 26.

9 Heidegger, Einführung in die phänomenologische Forschung, GA Bd. 17, Frankfurt a. M. 1994, S. 21. $\mathrm{Zu} » A n s p r e c h e n "$ und »Angesprochensein « bei Heidegger siehe E. S. Nelson, Ansprechen und Auseinandersetzung: Heidegger und die Frage nach der Vereinzelung von Dasein, in: Existentia 10 (2000), S. $113-122$. 
Doch die menschliche Existenz kann normaleweise nicht einfach mit sich selbst sprechen und sich selbst verstehen. Die Hermeneutik sollte sich deswegen »destruktiv« gegen die verfremdete Begrifflichkeit der Überlieferung wenden, um das Leben in seiner vollen, immanenten und weltlichen Tatsächlichkeit auszulegen, seine Fraglichkeit herauszustellen und es stets noch ursprünglicher zu deuten. ${ }^{10}$

Obwohl Heidegger genau diesen Denkweg zum Teil in Auseinandersetzung mit Diltheys Philosophie entwickelt, ging er Mitte der 1920er Jahre von der Hermeneutik des faktischen Lebens zur Ontologie des Seins über: zur »ersten Anzeige der Faktizität«. Heidegger verwendet die »Lebensphilosophie«, um zur Ontologie zu gelangen, die der Lebensbefangenheit entreißen soll. ${ }^{11}$ Das $»$ Wie» (die Art und Weise des Umgangs oder die Methode) und das "Was« (die Inhalte oder die Sachen selbst) sollten nach Heidegger aus der begrenzten Bedingtheit die er bei Dilthey kritisierte - herausgelöst, verallgemeinert und formalisiert werden. $^{12}$

Die Hermeneutik ist keine Art induktiver Erkenntnis, die vom Partikularen zum Allgemeinen aufsteigt, meint Heidegger, und damit wendet er sich gegen das Bild, das er von Dilthey hat. Diltheys Student und Schwiegersohn Georg Misch hat in seiner Abhandlung Lebensphilosophie und Phänomenologie diese Kritik beantwortet, indem er eine Art hermeneutischer Induktion verteidigt und den abstrakten »Deduktivismus « und den Primat der theoretischen Philosophie in Heideggers Begriff der formalen Anzeige, den er schon vor Sein und Zeit aus Natorps Bericht kennengelernt hatte, kritisiert. ${ }^{13}$

Die Bezeichnung "formale Anzeige«, der Grundsinn aller philosophischen Begriffe, hat Heidegger bald durch den Begriff des "Weges " ersetzt. Aber in dieser Zeit soll der anzeigende erwartende Vorgriff, „das Wie des Worauf des Bezugs «, das auf keinen Inhalt reduziert werden kann, die Dinge selbst in ihrer Konkretheit befreien und sehen lassen. Heidegger hat dies zweimal gesagt, das erste Mal im Zusammenhang mit der »formalen Anzeige« und das zweite Mal in Sein und Zeit: Man müsse »das, was sich zeigt, so wie es sich von ihm selbst her zeigt, von ihm selbst her sehen lassen. ${ }^{14}$

10 Heidegger, Phänomenologische Interpretationen, S. 11.

11 Misch, Lebensphilosophie, S. 11.

12 Nach Heidegger sollten wir in der Frage das "was« als das Erfragte, das Gefragte und das Befragte unterscheiden. Heidegger, Prolegomena zur Geschichte des Zeitbegriffs, GA Bd. 20, hg. Petra Jaeger, Frankfurt a. M. 1979, S. 195.

13 Misch, Lebensphilosophie, S. 27.

14 Heidegger, Phänomenologische Interpretationen zu Aristoteles GA 61, S. 53; ders., Sein und Zeit, Tübingen 1985, S. 34. 


\section{Die hermeneutische Lebensphilosophie als Frage nach dem faktischen Leben}

Im Licht von Georg Mischs Lebensphilosophie und Phänomenologie sind die Differenzen zwischen Heidegger und Dilthey klarer zu deuten. Bei der Frage nach dem faktischen Leben geht es Heidegger nicht nur - wie in der Deutung Diltheys - um ein biologisches, anthropologisches und gemeinschaftliches Lebenskonzept und auch nicht um ein persönliches Leben. Ungeachtet der "Jemeinigkeit« des Lebens und des Todes kann Heidegger behaupten, dass Aristoteles gelebt hat und gestorben ist: d.h. dass die Philosophie von dem empirischen Leben des Aristoteles abgesondert wird. ${ }^{15}$ Dilthey aber interessiert sich für das singuläre Leben im Kontext der mehr oder weniger stabilen äußeren Bedingungen und gesellschaftliche Strukturen, und dies bedeutet, dass sein Lebensbegriff, der nicht nur abstrakt und allgemein funktioniert, auch im Lichte der persönlichen Biographie und Autobiographie zu verstehen ist. ${ }^{16}$

Heiddeger hingegen geht es hauptsächlich um einen Begriff des Lebens, das, wie Misch betont, immer abstrakter zu fassen ist. Durch die steigende Formalisierung des Lebens zu einem formal angezeigten Dasein wird das Dasein bei Heidegger von der sog. »Lebensphilosophie« befreit, die Dilthey noch gefangen hält. In diesem Zusammenhang kritisiert Heidegger die »Tautologien der Lebensphilosophie" und ihre Tatsächlichkeiten "von verschiedener Provenienz«, die für Dilthey jeweils nicht ableitbar aus einer anderen sind. Heidegger betont die Einheit des Seins gegenüber der Heterogenität bei Dilthey, und damit wird das "ontologisch" gefasste Leben des Daseins von allen "ontischen", biologischen sowie anthropologischen, Elementen befreit. ${ }^{17}$

In Heideggers Kritik ist Diltheys Denken den Aporien der Lebensphilosophie verfallen; aber dieses Denken zeige zugleich einen Ausweg auf, indem Dilthey die »Kategorien des Lebens« dargestellt habe. Heidegger selbst nutzte in seinen Vorlesungen von 1921/22 diese Terminologie der "Lebenskategorien", die er in Auseinandersetzung mit Dilthey entwickelt hatte, und erklärt, dass der Logos dem Leben und seiner antwortenden Selbst-Thematisierung wie auch der dem

15 Heidegger, Grundbegriffe der aristotelischen Philosophie, GA Bd. 18, Frankfurt a. M. 2002, S. $4 \mathrm{f}$.

$16 \mathrm{Zu}$ »ein Leben« und die hermeneutischen Konsequenzen des biographischen Lebens bei Dilthey siehe E. S. Nelson, Self-Reflection, Interpretation, and Historical Life in Dilthey, in: Recent Contributions to Dilthey's Philosophy of the Human Sciences, hg. von Hans-Ulrich Lessing, Rudolf A. Makkreel, Riccardo Pozzo, Stuttgart 2011, S. 105-134.

17 Dilthey, Einleitung in die Geisteswissenschaften, GS Bd. 1, S. 10; Heidegger, Einleitung in die Philosophie, GA Bd. 27, Frankfurt a. M. 2001, S. 347. 
Leben inhärenten Tendenz, sich selbst auszuweichen, sich selbst fern zu sein und sich selbst zu ruinieren, innewohnt. ${ }^{18}$

Die Lebensphilosophie, so behauptet Heidegger, hat diese Ruinanz und die Unheimlichkeit des Lebens unterschätzt, denn die Lebensphilosophie konnte die fundamentale Unruhe der Geschichte und des Lebens ${ }^{19}$, d.h. ihre immanente Fragwürdigkeit und die Tendenz, sich selbst zu ruinieren, nicht thematisieren. ${ }^{20}$ Aber das Leben wird nicht nur als Stabilität, Sicherheit und Gewissheit, sondern auch als Zerstreuung, Ferne, und Ruinanz erfahren. ${ }^{21}$ Obwohl schon Dilthey versuchte, eine logische Artikulation des Lebens in den »Kategorien des Lebens « zu entwickeln, behauptet Heidegger, dass dieser Versuch unzureichend war, insofern Dilthey die fundamentale Unruhe des Lebens und dessen Kategorien nur so weit akzeptierte, als er sie beruhigen und sublimieren konnte. ${ }^{22}$ Das Hin und Her in der Unruhe des Lebens zeigt die fundamentale geschehende Bewegtheit des Daseins. ${ }^{23}$ Diese Ruinanz des faktischen Lebens wird wenige Jahre später in Sein und Zeit in die Verfallenheit des Daseins umgedeutet: Das Verfallen zeigt sich als »eine existenzial eigene Weise der Bewegtheit. ${ }^{24}$

Laut Heidegger hat die Lebensphilosophie übersehen, dass das ständige "Leben-zum-Tode» - eine lang dauernde Geburt in der Faktizität der Sterblichkeit - eine Grundtendenz des menschlichen Daseins bezeichnet, nämlich das $\mathrm{Zu}-$ Grunde-Gehen. Es ist die Selbstaneignung des Lebens in seiner faktischen Zeitlichkeit sich selbst verfallen und ruiniert zu sein: "Das faktische, ruinante Leben `hat keine Zeit`, weil seine Grundbewegtheit, die Ruinanz selbst, die `Zeit wegnimmt, eine wegnehmbare Zeit, die faktisch ruinantes Lebens für sich selbst in sich selbst wegnimmt. ${ }^{25}$

Heideggers Begriff der Faktizität des Daseins ist also als Bedingung, Begrenzung und als Lebensfrage dieser Existenz zu verstehen. Jedoch hieß es bereits bei Dilthey, dass die Durchsichtigkeit des Selbst »den Gestalten des Dichters, nicht der Anschauung des wirklichen Lebens (eigne). ${ }^{26}$ Ebenso hat Dilthey in seiner späteren Kritik an Hegels Begriff des objektiven Geistes die Relativität und Vergänglichkeit, die Endlichkeit und Zerbrechlichkeit des menschlichen Daseins hervorgehoben:

18 Heidegger, Phänomenologische Interpretationen zu Aristoteles, GA Bd. 61, Kap. 2.

19 Heidegger, Phänomenologie des religiösen Lebens, GA Bd. 60, Frankfurt a. M. 1995, S. 30 - 54.

20 Heidegger, Phänomenologische Interpretationen zu Aristoteles, GA Bd. 61, S. 2.

21 Ebd., S. 103.

22 Heidegger, Phänomenologie des religiösen Lebens, GA Bd. 60, S. 38-50.

23 Heidegger, Phänomenologische Interpretationen zu Aristoteles, GA Bd. 61, S. 93.

24 Heidegger, Sein und Zeit, S. 134.

25 Heidegger, Phänomenologische Interpretationen zu Aristoteles, GA Bd. 61, S. 140.

26 Dilthey, Einleitung, GS Bd. 1, S. 62. 
»Hegel konstruiert metaphysisch; wir analysieren das Gegebene. Und die heutige Analyse der menschlichen Existenz erfüllt uns alle mit dem Gefühl der Gebrechlichkeit, der Macht des dunklen Triebes, des Leidens an der Dunkelheit und den Illusionen, der Endlichkeit in allem, was Leben ist, auch wo die höchsten Gebilde des Gemeinschaftslebens aus ihm entstehen. So können wir den objektiven Geist nicht aus der Vernunft verstehen, sondern müssen auf den Strukturzusammenhang der Lebenseinheiten, der sich in den Gemeinschaften fortsetzt, zurückgehen. ${ }^{27}$

Heidegger betont, dass die Lebensphilosophie dem Leben selbst, das sie erhellen und verstehen sollte, verfallen sei. Gegen die Weltbefangenheit stellt Heidegger die Radikalität der Entscheidung. ${ }^{28}$ Die Lebensphilosophie gründet für Heidegger deswegen nicht in einer erhellenden und geklärten Leidenschaft, sondern in einer Leidenschaft dem Prinzip gegenüber, das sie klären sollte. So kann sie sich selbst nicht zu Erhellung und Reife bringen. Sie ist wie eine »Botanik der Pflanzen« eine leere Tautologie, die nichts über den kategorialen und formalen Charakter des Lebens, welches sie thematisiert, zu sagen hat. ${ }^{29}$ Sie sagt nichts über den ontologischen Rang des Lebens ${ }^{30}$, d.h. über den formalen aber nicht logischen Charakter des Lebens, den sie auf Grund der "Kategorien des Lebens» oder existentiell begreifen sollte.

Die formalisierende Aufhebung der ontischen empirischen Mannigfaltigkeit des Lebens, die sogenannte Blindheit der Partikularitäten, die Heidegger gegen Dilthey auch in der Vorlesung "Einleitung in die Philosophie« vom Wintersemester 1928/29 behauptet, bedeutet die Möglichkeit, die Einheit des Sein-Sinnes in der "ontologischen Differenz« von Sein und Seiendem zu denken. Beides, die Einheit und die Differenz, geschehen jenseits der »heterogenen « Unterschiede von Natur und Geschichte und jenseits der Differenzen der Weltanschauungen usw., die bei Dilthey von unableitbarer, »verschiedener Provenienz« sind.

Heideggers Analytik des Daseins wollte deswegen nur eine neutrale vor-anthropologische Grundverfassung der Existenz beschreiben. Wegen der faktischen Zerstreuung in der Welt ist das Dasein wesentlich ein gebrochenes Neutrum. ${ }^{31}$ Heidegger betont: "Dieses neutrale Dasein ist nie das Existierende; es existiert das Dasein je nur in seiner faktischen Konkretion. ${ }^{32}$ Vielmehr geht das neutrale Dasein stets der Leiblichkeit, Geschlechtlichkeit sowie den anderen ontischen Eigenschaften der Menschen voraus. ${ }^{33}$ In diesem Kontext ist die

27 Dilthey, Der Aufbau der geschichtlichen Welt in den Geisteswissenschaften, GS Bd. 7, S. 150.

28 Misch, Lebensphilosophie, S. 7.

29 M. Heidegger, Logik. Die Frage nach der Wahrheit, Frankfurt a. M. 1976, S. 216.

30 Heidegger, Sein und Zeit, S. 46.

31 Heidegger, Einleitung in die Philosophie GA Bd. 27, S. 146.

32 M. Heidegger, Metaphysische Anfangsgründe der Logik im Ausgang von Leibniz, GA Bd. 26, Frankfurt a. M. 1990, S. 173.

33 Heidegger, Metaphysische Anfangsgründe, GA Bd. 26, S. 172. 
menschliche Existenz als das jedesmal eigene Dasein in seinem Seinsbezug zu verstehen: d.h. als jeweils mein eigenen Daseins (als "Jemeinigkeit«) im Verhältnis zu dem unpersönlichen $>$ Da $<$ des Seins. ${ }^{34}$

Misch beschrieb in Lebensphilosophie und Phänomenologie, wie Heidegger »ethisch-idealistisch eingestellt ist, während bei Dilthey die objektiv-idealistische Einstellung sich darin verriete." Misch betont gleichzeitig die Übermacht der Strukturen und der unpersönlichen Tendenzen der ontologischen Wende Heideggers. ${ }^{35}$ Im Unterschied zu Diltheys Sich-Finden von Selbst und Anderen in »einer Atmosphäre von Gemeinsamkeit« hat Heidegger nach Misch die »radikalste Individuation « verabsolutiert und verdinglicht. ${ }^{36}$

Interessanterweise hat Rudolf Carnap die Metaphysik als eine Verdinglichung des Lebens kritisiert, und später hat Emmanuel Lévinas, der Heideggers Vorlesung im Wintersemester 1928/29 besuchte, das unpersönliche Sein bei Heidegger - das »es gibt« (il y $a$ ) - als ein Sein beschrieben, das von jeder zwischenmenschlichen Begegnung und somit auch von ethischen Beziehungen losgelöst ist. ${ }^{37}$

\section{Dilthey: Eine Alternative zur ontologischen Wende Heideggers?}

Obwohl Heidegger einige Grundbegriffe und Strategien aus dem Begriffsfeld von Dilthey übernommen hat, wie wir gesehen haben, gibt es bestimmte unüberwindbare Unterschiede zwischen Diltheys hermeneutischer Philosophie der Wissenschaften und seiner Philosophie des Lebens einerseits und der philosophischen, ontologischen Hermeneutik Heideggers andererseits.

Im Begriff der Zeit von 1924 hat Heidegger sich auf Diltheys Freund Graf Yorck von Wartenburg bezogen: "Yorck findet, dass Diltheys Untersuchungen >zu wenig die generische Differenz zwischen Ontischem und Historischem betonen, « und er meinte damit die Differenz zwischen dem »okularen« oder optischen Schauspiel, das historisch beschrieben und verglichen wird, und der lebendigen Virtualität der Geschichtlichkeit, die ursprünglich-geschichtlich gelebt wird. Die Virtualität bedeutet das Möglichsein des Daseins, das sich primär aus seiner Zukunft versteht.

Die Hauptgründe für den Unterschied zwischen Diltheys Philosophie und

34 Heidegger, Einleitung in die Philosophie, GA Bd. 27, S. 347.

35 Misch, Lebensphilosophie, S. 29-30.

36 Ebd., S. $82-83$.

37 Rudolf Carnap, Scheinprobleme in der Philosophie und andere metaphysikkritische Schriften, Hamburg 2004, S. 51; Emmanuel Lévinas, Die Zeit und der Andere, Hamburg 2003, S 24. 
Heideggers Daseinsanalytik (und der ontologischen Hermeneutik von Yorck bis Gadamer) hat teilweise schon Misch genannt, es sind die folgenden:

(1) Dilthey zielte ab auf eine objektive wissenschaftlich orientierte Philosophie, eine Philosophie, die die Wissenschaften und ihre Vernunft erweitert und ihre Aufgabe in der menschlichen Welt versteht.

(2) Die Natur und die Naturwissenschaften, die Heidegger grundsätzlich in Frage stellt, die bei Dilthey aber eine positive Rolle spielen ${ }^{38}$, wollte Dilthey kontextualisieren und nur begrenzen, ohne ihre eigene Bedeutung aufzuheben.

(3) Die wissenschaftliche Forschung benötigt laut Dilthey die ontische Dynamik des Lebens, die empirische Heterogenität, die widerstrebende Materialität der Sachen selbst, welche die beschreibenden, induktiven, reflektierenden und komparativen Methoden erzeugt. Diese Dynamik spricht gegen Heideggers umgreifende Einheit und monistische Ganzheit des Sinns des Seins, die Misch als eine radikale allgemeine Dynamik ohne geschichtliches und wirkendes Werden beschreibt. ${ }^{39}$ Misch merkt weiter an, wie bei Heidegger alle Seinsweisen des Daseins die theoretische Einheit des Seins voraussetzen und die Fragen der Wissenschaften und des praktischen Lebens nur als »Sonderprobleme « verstanden werden. ${ }^{40}$

(4) Dilthey, in diesem Zusammenhang eher W. von Humboldt und Herder näher als Hegel, hat die Bedeutsamkeit und den Wert der kulturellen und individuellen Unterschiede gegenüber der Einheit der kulturellen Überlieferung verstanden und betont.

(5) Schließlich wollte Dilthey mit seinem weltimmanenten Personalismus den Sinn und die Werte des persönlichen und ethischen Lebens des Individuums mitten in ihrer Kontextabhängigkeiten von natürlichen Gewalten, von geschichtlichen Prozesse und sozialen Strukturen deuten und verteidigen.

Von diesem Standpunkt aus, der von Yorck bis Heidegger und Gadamer als "Kulturliberalismus« bezeichnet wird, bleibt Dilthey ein Philosoph der Moderne. ${ }^{41}$ Misch betont die Umwandlung und Fortsetzung der Aufklärung in einer geschichtlichen, lebensphilosophischen und pluralistischen Bildungsphilosophie bei Dilthey; ${ }^{42}$ dennoch bleibt Diltheys Stellung ambivalent: Er ist ein Ver-

38 Vgl. Misch, Lebensphilosophie, S. 23, 33.

39 Ebd., S. 43.

40 Ebd., S. 10-14, 35.

41 Hans-Georg Gadamer, Hermeneutik im Rückblick. Gesammelte Werke Bd. 10, Tübingen 1995, S. 9.

42 Vgl. Georg Misch, Von den Gestaltungen der Persönlichkeit, in: Weltanschauung. Philosophie und Religion in Darstellungen von Wilhelm Dilthey u.a. [hg. von Max Frischeisen-Köhler], 
treter der Lebensphilosophie und der Aufklärung, und zwar in ihrer geschichtlich erweiterten und vertieften Gestalt; das ergibt sich z. B. klar aus den letzten Passagen seines Werkes Das Wesen der Philosophie. Die lebensphilosophische Nähe zu Gefühl und Leben sollte nicht in ihrer Unmittelbarkeit verbleiben, sondern in der Selbstbesinnung methodisch reflektiert und geprüft werden. ${ }^{43}$

Allerdings hat Diltheys reflektierende prüfende Besonnenheit des Lebens aus und über sich selbst nicht den Charakter von Heideggers Formalisierung und Entleerung des Daseins, weil die Selbstbesinnung und Selbstmitteilung bei Dilthey nicht so deutlich von den konkreten Lebensinhalten und Verweisungszusammenhängen abstrahiert werden können. Das gilt auch für die empirische, induktive und komparative Erforschungen der »Tatsächlichkeit» (s. u.), die nicht nur im Denken als ein Faktum des Daseins gegeben ist.

Heideggers Forderung, „den Geist des Grafen Yorck zu pflegen, um dem Werke Diltheys zu dienen«, bedeutet eine Umkehrung, die etwas Wichtiges vergisst und verfehlt, nämlich die empirische Mannigfaltigkeit und die zwischenmenschliche Verbindlichkeit des gemeinsamen Lebens innerhalb des Seins. ${ }^{44}$ Insofern ist Diltheys Verfahrensweise für das Verständnis des geschichtlichen, praktischen Lebens zwischen den Standpunkten der ontologischen Immanenz des Seins und der ethischen Transzendenz zu positionieren. Sie vermittelt das individuell persönliche Moment, das innerhalb der faktischen Immanenz der natürlichen und gesellschaftlichen Bedingungen und Begegnungen angesiedelt und bedeutsam ist.

\section{Die Wände der Tatsächlichkeit und die Endlichkeit des biographischen Lebens}

Dilthey hat folgendes ausgesprochen: „Der letzte Erklärungsgrund der Welt ist die Tatsächlichkeit. ${ }^{45}$ Die Tatsächlichkeit ist nicht eine »tote äußere Tatsächlichkeit«, sondern in der inneren Erfahrung und im Erlebnis gegeben und lebendig. Das bloß Faktische wird im Innewerden, im Lebensgefühl und in der Besinnung als eine gegenübertretende Wirklichkeit erfahren, die »da für mich» ist.

Das »da für mich« und die Tatsächlichkeit des Bewusstseins sind weitere

Berlin 1911, S. 82, 95; und auch E. S. Nelson, Heidegger, Misch and the Origins of Philosophy, in: Journal of Chinese Philosophy 39, Supplemental Issue (2012), S. 41.

43 Dilthey, GS Bd. 5, S. 412.

44 Gadamer, Hermeneutik im Rückblick, S. 9.

45 Dilthey, Der Aufbau, GS Bd. 7, S. 53. 
Phänomena als die Tatsachen der physikalischen Welt und des gesellschaftlichen Lebens. Es geht hier um eine reflexive Tatsächlichkeit, die Heidegger eher die Faktizität nennen würde, es geht um das Verhältnis des Selbst zu sich selbst ohne einen bestimmten Bezug oder einen Gegenstand. So kann z. B. ein Gefühl eine Stimmung des eigenen Selbst sein, die nur in dem selbstbezogenen Innewerden existiert: »Wenn ich mich traurig fühle, so ist dies Gefühl von Traurigkeit nicht mein Objekt, sondern indem dieser Zustand mir bewußt ist, ist er für mich da, für mich, als welchem er eben bewußt ist. Ich werde seiner inne. $\aleph^{46}$

Dieses »da sein für mich« ist nicht nur als Gefühl und affektives Leben gegeben, da es ferner die Möglichkeit bedeutet, eine Welt zu haben, ein Thema, das Heidegger von Dilthey aufgenommen hat. Nach Dilthey ist die Materialität als eine Art der Tatsächlichkeit zu deuten, als eine Mannigfaltigkeit, die nicht verneint oder aufgehoben werden kann. Aber die Tatsächlichkeit umfasst mehr als die physikalischen Elemente und die Momente dieser Mannigfaltigkeit, die nur im Widerstand und in der Auseinandersetzung, d.h. in der Differenzierung von Selbst und Welt, zu einer oder der "Welt» wird. Die »Welt» ist in diesem Sinn nicht nur ein ontologisches »es weltet«, das unabhängig von den ontischen Unterschieden, die eine Welt konstituieren, geschehen kann.

Das menschliche Selbst existiert für Dilthey nie ohne die Andersheit und Weltlichkeit, deren Widerstand es ausgesetzt ist. ${ }^{47}$ Heidegger hat Diltheys Deutung des Widerstands in Sein und Zeit kritisiert, weil Widerstand sowie Leiblichkeit schon das In-der-Welt-sein und die Welt voraussetzen. ${ }^{48}$ Schon in den Prolegomena zur Geschichte des Zeitbegriffs vom Sommersemester 1925 argumentiert Heidegger: »[...] Widerstand [ist] ein phänomenaler Charakter, der Welt schon voraussetzt. ${ }^{49}$ Laut Dilthey aber ist es nicht die Einheit der Welt oder des Seins der Ontologie, sondern die Entzweiung zwischen Leib und Welt, NichtIch und Ich, Selbst und Anderen, die sinngebend ist für ein sinnhaftes Leben innerhalb der konkreten Spannungen, Oszillationen und Durchkreuzungen: »[...] nur ideale Repräsentation ist harmonisch. Jede reale Darstellung enthält ein Gegensätzliches von Gewahren der Singularität «. ${ }^{50}$

Die Biographie ist für Dilthey die höchste philosophische Form der Geschichte. ${ }^{51}$ Jedoch ist den Einzelnen in seinen verschiedenen Äußerungen zu verstehen und zu deuten eine unendliche Aufgabe, weil das Individuum unergründlich ist. Das Motto von Diltheys umfangreicher Biographie von Friedrich Schleiermacher war Goethes »individuum est ineffabile». Die Biographie ist eine

46 Dilthey, Ideen über beschreibende und zergliedernde Psychologie, GS Bd. 5, S. 197.

47 Vgl. Dilthey, Weltanschauungslehre, GS Bd. 8, S. 18.

48 Heidegger, Sein und Zeit, S. 209-211.

49 Heidegger, Prolegomena zur Geschichte des Zeitbegriffs, GA Bd. 20, S. 301.

50 Dilthey, Der Aufbau, GS Bd. 7, S. 331.

51 Dilthey, Ideen über beschreibende und zergliedernde Psychologie, GS Bd. 5, S. 225. 
kreisende Annäherung und Darstellung des Singularen, das weder eine pure irrationale Singularität ist noch vollständig begrifflich erfasst und vorgestellt werden kann und deshalb nicht ganz darstellbar ist. ${ }^{52}$

Es kann keineswegs in der Lebenserfahrung ein reines Chaos von ungedeuteten sinnfreien Singularitäten als facta bruta geben. Die begrifflichen Kategorien wie Ähnlichkeit und Identität, womit wir das Erfahrene ordnen, sind deswegen keine bloßen Illusionen; vielmehr bilden und entwickeln sich jene Kategorien in der Immanenz ihrer Lebenszusammenhänge, weil das Leben sinnschaffend ist und Sinndeutungen aufbaut. Die realen Lebenskategorien wie Selbigkeit und Differenz, Leiden und Wirken, Bedeutsamkeit sind in der weltlichen Dynamik begründet und haben schon in den elementarsten Regungen des Lebens ihre reale Funktion.

Das Selbst ist nicht als eine einfache Einheit oder als eine Person intuitiv oder unmittelbar sich selbst gegeben; das Individuum als Person ist gesellschaftlichgeschichtlich vermittelt, es wird mittelbar so verstanden und reflektierend so gedeutet; der Forderung nach der prinzipiellen Möglichkeit einer absoluten Gegebenheit kann nicht entsprochen und eine solche Möglichkeit kann nicht demonstriert werden. Das Leben ist für Dilthey nicht allein als ein subjektloses "es ist " oder als ein unpersönliches Ereignis zu schildern. ${ }^{53}$ Heideggers »jemeiniges« Dasein hingegen darf nicht als die jeweilige Biographie eines konkreten Menschen verstanden werden. Die Rede vom »Dasein « ist bei Heidegger nur formal-anzeigend und entleert von allen bestimmten Lebensinhalten.

Dilthey teilt deshalb auch nicht Heideggers Abneigung gegenüber historischen biographischen Darstellungen des Lebens. Ein eigenes Leben ist nicht nur ein neutrales Ereignis und Geschehen; es wird erlebt und durchlebt, gebildet und ausgedrückt, verstanden und ausgelegt. Diltheys hermeneutischer Dreischritt von elementarem und komplexem Erleben, Ausdruck und Verstehen fungiert wie ein dynamischer Wirkungszusammenhang. Im jeweiligen gesellschaftlichen geschichtlichen Kontext von Gewalt, Strukturen und Verweisungen ist der Mensch als Person durch mannigfaltige Bildungs- und Lernprozesse vereinzelt.

Zwischen dem Selbst und den Anderen, in der Selbst-Distanz und SelbstFremdheit der Selbststellung und in der innewerdenden Reflexivität und Besinnung vollzieht sich ein Leben. Ein »Leben als Ganzes" geschieht in der lebensweltlichen praktischen Ausbildung einer lebensgeschichtlichen ethischen Einheit, die nicht wie bei Heidegger auf eine radikale Entscheidung oder Entschlossenheit zurückführbar ist. Dieses individuelle Leben ist eher als eine

52 Vgl. E. S. Nelson, Begründbarkeit und Unergründlichkeit bei Wilhelm Dilthey, in: Existentia 12/1-2 (2002), S. 9.

53 Vgl. Emmanuel Lévinas, Humanismus des anderen Menschen, Hamburg 2005, S. 138. 
ethische Gestaltung und Bildung denn als eine romantische Fiktion zu verstehen.

In Helmuth Plessners anthropologischer Deutung heißt es: "Der Mensch lebt nur, indem er ein Leben führt.. ${ }^{54}$ Das Leben ist zugleich biologisch-geschichtlich er- und ge-lebt: ein Leben geschieht in seinem interpretativen Vollzug, in der Auslegung und Deutung des Lebens. ${ }^{55}$ In diesem Zusammenhang bezeichnet Plessner Diltheys Philosophie als eine "neue Anthropologie — im Sinne einer ethisch-politisch orientierten geschichtlichen Besinnung eines Lebens - und betont überdies die ethische-soziale Bedeutsamkeit der politischen Anthropologie: die Politik, wenn sie nicht nur blinde Macht ist, sei der "geschichtsaufschließende Horizont eines Lebens, das sich zur ganzen Welt ermächtigt weiß «. ${ }^{56}$

Bei Dilthey und Plessner gibt es anders als bei Heidegger eine enge und konstitutive Beziehung zwischen der allgemeinen Anthropologie des Lebens und der Autobiographie eines bestimmten persönlichen Lebens, die eine biographische Anthropologie des »exzentrischen" individualisierten Menschen ermöglicht. Das Sich-selbst-verstehen-Lernen, die Selbstbeziehung und die elementare Selbstbesinnung in der Autobiographie als eine hermeneutisch darstellende Kunst sind deswegen ebenfalls keine unmittelbare Selbstanschauung von einem allgemeinen Selbst oder eine Enthüllung vom »Dasein im Menschen. ${ }^{57}$

Während in Heideggers Ontologie das Dasein im Menschen ontologisch weltbildend ist, ist weltbildend in Diltheys geschichtlichem Denken eher das faktische autobiographische Leben. Dieses Leben ist entweder ein singulares »ich« oder ein plurales »wir«, ohne ein kollektives Subjekt darzustellen. Die Autobiographie ist eine interpretative lebensgeschichtliche Selbstauslegung im Kontext der Selbstbildung, die mit der grundlegenden konstitutiven Rolle der Einbildungskraft im Leben in Verbindung steht; diese Verbindung besteht wegen der beständigen Vermittlung und der Endlichkeit meines faktischen individuellen Bewusstseins und persönlichen Lebens.

54 Helmuth Plessner, Die Stufen des Organischen und der Mensch. Einleitung in die Philosophische Anthropologie, Berlin 1975, S. 310, 345.

55 Vgl. Helmuth Plessner, Macht und menschliche Natur, GS Bd. 5, Frankfurt a. M. 1981, S. 231.

56 Ebd., S. 165, 220. Vgl. Volker Schürmann, Die Unergründlichkeit des Lebens. Lebens-Politik zwischen Biomacht und Kulturkritik, Bielefeld 2011.

57 Heidegger, Die Grundbegriffe der Metaphysik, GA Bd. 29/30, Frankfurt a. M. 1992, S. 414; vgl. Otto Friedrich Bollnow, Dilthey. Eine Einführung in seine Philosophie, Stuttgart, Berlin, Köln, Mainz 1967, S. 45. 


\section{Abschluss}

Dilthey hat folgendes bemerkt: "Der Widerstand wird zum Druck, ringsum scheinen uns Wände von Tatsächlichkeit zu umgeben, die wir nicht durchbrechen können«. »Immer sind Wände da. ${ }^{58}$ In Otto Friedrich Bollnows DiltheyDeutung ist das menschliche Verhältnis zur Wirklichkeit so, dass der Mensch sie als eine »drohenden Macht« erfährt, »die den Menschen umklammert hält und der er ausgeliefert ist. ${ }^{59}$

Die lebensbezogene Faktizität, deren Ursprung jenseits des abstrakten begrifflichen Denkens liegt und deren Wert und Geltung seiner Macht entzogen bleibt, ist durch Widerstand und Druck in der Differenzierung des Selbst und der Welt gegeben und ermöglicht den Umfang und die Grenzen des Aufbaus der natürlichen und sozialen Objektivität. Dilthey legt dar, wie die Faktizität der letzte Grund der Erkenntnis ist, weil die Erkenntnis die Faktizität nicht durchdringen kann, um die Faktizität und die Singularität innerhalb des unergründlichen gestaltenden Zusammenhangs des Lebens zu deuten. ${ }^{60}$

Nach Diltheys Argumentation ist Wissen und Erkennen möglich, aber immer nur lebensimmanent und kontextuell: »Hinter das Leben kann das Erkennen nicht zurückgehen, d.h. es kann keinen Zusammenhang machen, der nicht in der eigenen Lebendigkeit gegeben ist. ${ }^{61}$ Dies bedeutet, dass die elementare Spannung und prinzipielle Unvergleichbarkeit zwischen dem unentwirrbaren Zusammenhang und dem Individuum, zwischen dem zusammenhängenden Ganzen und dem Singularen, nicht zu Ende kommen kann.

58 Dilthey, Beiträge zur Lösung der Frage vom Ursprung unseres Glaubens an die Realität der Außenwelt und seinem Recht (1890), GS Bd. 5, S. 105.

59 Bollnow, Dilthey, S. 32.

60 Dilthey, Einleitung, GS Bd. 1, S. 322; Leben Schleiermachers, GS Bd. 13, S. 53.

61 Dilthey, Weltanschauungslehre, GS Bd. 8, S. 180. 
